\title{
On Some Predictive Ratio Type Estimators in Two Stage Sampling
}

\author{
A.K.P.C. Swain ${ }^{1}$, Sitanshu Shekhar Mishra ${ }^{2}$, and B.K. Pradhan ${ }^{3}$ \\ ${ }^{1}$ Former Professor of Statistics, Utkal University, Bhubaneswar-751004, Odisha, India \\ ${ }^{2}$ Lecturer in Statistics, Trident Academy of Creative Technology (TACT), Bhubaneswar, Odisha, India \\ ${ }^{3}$ Former Reader in Statistics, Utkal University, Bhubaneswar-751004, India \\ akpcs@rediffmail.com
}

Received 10 March 2012

Accepted 13 January 2013

\begin{abstract}
Some predictive ratio type estimators in two stage sampling are derived following the model free predictive criterion proposed by Basu (1971) and these estimators are compared with the classical multistage ratio estimator and also with Smith's (1969) multistage ratio estimator. A numerical illustration is provided to compare the efficiencies of different predictive estimators.
\end{abstract}

Keywords: Two stage sampling, Predictive estimator, Ratio Estimator.

AMS Classification: 62D05.

\section{Introduction}

Let a finite population $U$ be partitioned into $N$ clusters of first stage units (f.s.u.) denoted by $\left(U_{1}, U_{2}, \ldots, U_{N}\right)$ such that the number of second stage units (s.s.u.) in $U_{i}$ be $M_{i}(i=1,2, \ldots, N)$. Further, let $y_{i j}$ and $x_{i j}$ be the values of the study variable $(y)$ and the auxiliary variable $(x)$ respectively corresponding to $j$ th s.s.u. in $i$ th f.s.u. $\left(i=1,2, \ldots, N ; j=1,2, \ldots, M_{i}\right)$.

Define

$$
\begin{gathered}
\bar{Y}_{i}=\frac{1}{M_{i}} \sum_{j=1}^{M_{i}} y_{i j}, \quad \bar{X}_{i}=\frac{1}{M_{i}} \sum_{j=1}^{M_{i}} x_{i j}, \quad Y=\sum_{i=1}^{N} \sum_{j=1}^{M_{i}} y_{i j}, \\
\bar{Y}=\frac{1}{N} \sum_{i=1}^{N} u_{i} \bar{Y}_{i}, \quad X=\sum_{i=1}^{N} \sum_{j=1}^{M_{i}} x_{i j}, \quad \text { and } \quad \bar{X}=\frac{1}{N} \sum_{i=1}^{N} u_{i} \bar{X}_{i},
\end{gathered}
$$

where $u_{i}=\frac{M_{i}}{\bar{M}}, \bar{M}=\frac{\sum_{i=1}^{N} M_{i}}{N}$.

Also, $R=\frac{\bar{Y}}{\bar{X}}$ and $R_{i}=\frac{\bar{Y}_{i}}{\bar{X}_{i}}(i=1,2, \ldots, N)$. 
Assume that a simple random sample (without replacement) $s$ of size $n$ f.s.u. is selected from $U$ and then at the second stage a sample $s_{i}$ of size $m_{i}$ s.s.u. from the $i$ th selected f.s.u. $U_{i}(i=1,2, \ldots, n)$ is selected according to simple random sampling without replacement.

Now define $\bar{y}_{i}=\frac{1}{m_{i}} \sum_{j=1}^{m_{i}} y_{i j}, \bar{x}_{i}=\frac{1}{m_{i}} \sum_{j=1}^{m_{i}} x_{i j}$.

Further define

$$
\begin{aligned}
S_{b y}^{2} & =\frac{1}{N-1} \sum_{i=1}^{N}\left(u_{i} \bar{Y}_{i}-\bar{Y}\right)^{2}, \\
S_{b x}^{2} & =\frac{1}{N-1} \sum_{i=1}^{N}\left(u_{i} \bar{X}_{i}-\bar{X}\right)^{2}, \\
S_{b y x} & =\frac{1}{N-1} \sum_{i=1}^{N}\left(u_{i} \bar{Y}_{i}-\bar{Y}\right)\left(u_{i} \bar{X}_{i}-\bar{X}\right), \\
\rho_{b y x} & =\frac{S_{b y x}}{S_{b y} S_{b x}}, \quad C_{b y}=\frac{S_{b y}}{\bar{Y}}, \quad C_{b x}=\frac{S_{b x}}{\bar{X}} .
\end{aligned}
$$

For each $U_{i}(i=1,2, \ldots, N)$, define

$$
\begin{aligned}
S_{i y}^{2} & =\frac{1}{M_{i}-1} \sum_{j=1}^{M_{i}}\left(y_{i j}-\bar{Y}_{i}\right)^{2}, \\
S_{i x}^{2} & =\frac{1}{M_{i}-1} \sum_{j=1}^{M_{i}}\left(x_{i j}-\bar{X}_{i}\right)^{2}, \\
S_{i y x} & =\frac{1}{M_{i}-1} \sum_{j=1}^{M_{i}}\left(y_{i j}-\bar{Y}_{i}\right)\left(x_{i j}-\bar{X}_{i}\right), \\
\rho_{i y x} & =\frac{S_{i y x}}{S_{i y} S_{i x}}, \quad C_{i y}=\frac{S_{i y}}{\bar{Y}_{i}}, \quad C_{i x}=\frac{S_{i x}}{\bar{X}_{i}}, \quad(i=1,2, \ldots, N) .
\end{aligned}
$$

For any given sample ' $s$ ' we write the total of $y$ as

$$
\begin{aligned}
Y & =\sum_{i=1}^{N} \sum_{j=1}^{M_{i}} y_{i j} \\
& =\sum_{i=1}^{n} \sum_{j=1}^{m_{i}} y_{i j}+\sum_{i=1}^{n} \sum_{j=m_{i}+1}^{M_{i}} y_{i j}+\sum_{i=n+1}^{N} \sum_{j=1}^{M_{i}} y_{i j} \\
& =\sum_{i \in s} \sum_{j \in s_{i}} y_{i j}+\sum_{i \in s} \sum_{j \in \bar{s}_{i}} y_{i j}+\sum_{i \in \bar{s}} \sum_{j \in U_{i}} y_{i j},
\end{aligned}
$$

where $s \cup \bar{s}=U$ and $s_{i} \cup \bar{s}_{i}=U_{i}$.

As the first term in (1.1) is already known from the observed sample, we predict only second and third terms in (1.1) by model free prediction approach of Basu (1971) using auxiliary information on $x$, to arrive at an estimate of $Y$.

Thus, we may predict each $y_{i j}, j \in \bar{s}_{i}$ using the ratio method by $T_{i} x_{i j}$ and each $y_{i j}, i \in \bar{s}$ and $j \in U_{i}$, using also the ratio method by $T x_{i j}$. It may be mentioned here that $T_{i}$ is an estimate of ratio $\frac{\bar{Y}_{i}}{\bar{X}_{i}}(i=1,2, \ldots, N)$ from the ith first stage sample and $T$ is an estimate of $\frac{\bar{Y}}{\bar{X}}$ from the entire sample. 
Different choices of $T_{i}$ and $T$ lead to different predictive estimators in two stage sampling.

In the following we examine some of these choices to derive predictive estimators and compare them with the non-predictive estimators used in practice.

\section{Predictive Estimators}

\section{Case I}

Let $T_{i}=\frac{\bar{y}_{i}}{\bar{x}_{i}}$ and $T=\frac{\sum_{i=1}^{n} u_{i} \bar{y}_{i}}{\sum_{i=1}^{n} u_{i} \bar{x}_{i}}$.

Then the Basu type model free predictive estimator of $\bar{Y}$ earlier suggested by Panda (1998) is given by

$$
t_{p 1}=\frac{\sum_{i=1}^{n} u_{i} \bar{y}_{i}}{\sum_{i=1}^{n} u_{1} \bar{x}_{i}} \bar{X}+f\left[\frac{1}{n} \sum_{i=1}^{n} u_{i} \bar{y}_{i} \bar{x}_{i}-\frac{\sum_{i=1}^{n} u_{i} \bar{y}_{i}}{\sum_{i=1}^{n} u_{i} \bar{x}_{i}} \frac{1}{n}\left(\sum_{i=1}^{n} u_{1} \bar{X}_{i}\right)\right]
$$

where $f=\frac{n}{N}$.

Bias of $t_{p 1}$ is o $\left(\frac{1}{n}\right)$. Neglecting the bias for large samples, the approximate mean square error (MSE) of $t_{p 1}$ is given by

$$
\begin{aligned}
\operatorname{MSE}\left(t_{p 1}\right)= & \left(\frac{1}{n}-\frac{1}{N}\right)\left(S_{b y}^{2}+R^{2} S_{b x}^{2}-2 R S_{b y x}\right) \\
& +\frac{1}{n N} \sum_{i=1}^{N} u_{i}^{2}\left(\frac{1}{m_{i}}-\frac{1}{M_{i}}\right)\left(S_{i y}^{2}+\Delta_{1 i}^{2} S_{i x}^{2}-2 \Delta_{1 i} S_{i x y}\right),
\end{aligned}
$$

where $\Delta_{1 i}=R-f\left(R-R_{i}\right), i=1,2, \ldots, N$.

\section{Case II}

Let $T_{i}=\frac{\bar{y}_{i}}{\bar{X}_{i}}$ and $T=\frac{\sum_{i=1}^{n} u_{i} \bar{y}_{i}}{\sum_{i=1}^{n} u_{i} \bar{x}_{i}}$.

The predictive estimator of $\bar{Y}$ is thus given by

$$
\begin{aligned}
t_{p 2}= & \frac{1}{N \bar{M}}\left[\sum_{i=1}^{n} m_{i} \bar{y}_{i}+\sum_{i=1}^{n} \frac{\bar{y}_{i}}{\bar{X}_{i}}\left(M_{i} \bar{X}_{i}-m_{i} \bar{x}_{i}\right)+\frac{\sum_{i=1}^{n} u_{i} \bar{y}_{i}}{\sum_{i=1}^{n} u_{i} \bar{x}_{i}}\left(X-\sum_{i=1}^{n} M_{i} \bar{X}_{i}\right)\right] \\
= & \frac{\sum_{i=1}^{n} u_{i} \bar{y}_{i}}{\sum_{i=1}^{n} u_{i} \bar{x}_{i}} \bar{X}+f\left[\frac{1}{n} \sum_{i=1}^{n} u_{i} \bar{y}_{i}+\frac{1}{n} \sum_{i=1}^{n} f_{i} u_{i}\left(\bar{y}_{i}-\frac{\bar{y}_{i} \bar{x}_{i}}{\bar{X}_{i}}\right)-\frac{\sum_{i=1}^{n} u_{i} \bar{y}_{i}}{\sum_{i=1}^{n} u_{i} \bar{x}_{i}} \cdot \frac{1}{n} \sum_{i=1}^{n} u_{i} \bar{X}_{i}\right],
\end{aligned}
$$

where $f_{i}=\frac{m_{i}}{M_{i}}(i=1,2, \ldots, n)$. 
To the first order of approximation

$$
\begin{aligned}
\operatorname{MSE}\left(t_{p 2}\right)= & \left(\frac{1}{n}-\frac{1}{N}\right)\left(S_{b y}^{2}+R^{2} S_{b x}^{2}-2 R S_{b y x}\right) \\
& +\frac{1}{n N} \sum_{i=1}^{N} u_{i}^{2}\left(\frac{1}{m_{i}}-\frac{1}{M_{i}}\right)\left(S_{i y}^{2}+\Delta_{2 i}^{2} S_{i x}^{2}-2 \Delta_{2 i} S_{i x y}\right),
\end{aligned}
$$

where $\Delta_{2 i}=R-f\left(R-f_{i} R_{i}\right), i=1,2, \ldots, N$.

\section{Case III}

Let $T_{i}=\frac{\bar{y}_{i}}{\bar{x}_{i}}$ and $T=\frac{\sum_{i=1}^{n} u_{i} \bar{y}_{i}}{\sum_{i=1}^{n} u_{i} \bar{X}_{i}}$.

In such a situation the predictive estimator of $\bar{Y}$ is given by

$$
t_{p 3}=\frac{\sum_{i=1}^{n} u_{i} \bar{y}_{i}}{\sum_{i=1}^{n} u_{i} \bar{X}_{i}} \bar{X}+f\left[\frac{1}{n} \sum_{i=1}^{n} u_{i} \bar{y}_{i} \bar{x}_{i}-\frac{1}{n} \sum_{i=1}^{n} u_{i} \bar{y}_{i}\right] .
$$

The mean square error of $t_{p 3}$ to o $\left(\frac{1}{n}\right)$ is given by

$$
\begin{aligned}
\operatorname{MSE}\left(t_{p 3}\right)= & \left(\frac{1}{n}-\frac{1}{N}\right)\left(S_{b y}^{2}+R^{2} S_{b x}^{2}-2 R S_{b y x}\right) \\
& +\frac{1}{n N} \sum_{i=1}^{N} u_{i}^{2}\left(\frac{1}{m_{i}}-\frac{1}{M_{i}}\right)\left(S_{i y}^{2}+\Delta_{3 i}^{2} S_{i x}^{2}-2 \Delta_{3 i} S_{i x y}\right),
\end{aligned}
$$

where $\Delta_{3 i}=f R_{i}, i=1,2, \ldots, N$.

\section{Case IV}

Let $T_{i}=\frac{\bar{y}_{i}}{\bar{X}_{i}}$ and $T=\frac{\sum_{i=1}^{n} u_{i} \bar{y}_{i}}{\sum_{i=1}^{n} u_{i} \bar{X}_{i}}$.

The predictive estimator of $\bar{Y}$ in such a situation takes the form

$$
t_{p 4}=\frac{\sum_{i=1}^{n} u_{i} \bar{y}_{i}}{\sum_{i=1}^{n} u_{i} \bar{X}_{i}} \bar{X}+\frac{1}{n} \sum_{i=1}^{n} f f_{i} u_{i}\left(\bar{y}_{i}-\frac{\bar{y}_{i} \bar{x}_{i}}{\bar{X}_{i}}\right) .
$$

To the first order of approximation

$$
\begin{aligned}
\operatorname{MSE}\left(t_{p 4}\right)= & \left(\frac{1}{n}-\frac{1}{N}\right)\left(S_{b y}^{2}+R^{2} S_{b x}^{2}-2 R S_{b y x}\right) \\
& +\frac{1}{n N} \sum_{i=1}^{N} u_{i}^{2}\left(\frac{1}{m_{i}}-\frac{1}{M_{i}}\right)\left(S_{i y}^{2}+\Delta_{4 i}^{2} S_{i x}^{2}-2 \Delta_{4 i} S_{i x y}\right),
\end{aligned}
$$

where $\Delta_{4 i}=f f_{i} R_{i}, i=1,2, \ldots, N$. 


\section{Case V}

Let $T_{i}=\frac{\bar{y}_{i}}{\bar{x}_{i}}$ and $T=\frac{\sum_{i=1}^{n} u_{i} \frac{\bar{y}_{i}}{x_{i}} \bar{X}_{i}}{\sum_{i=1}^{n} u_{i} \bar{X}_{i}}$.

The predictive estimator of $\bar{Y}$ in this case is

$$
t_{p 5}=\frac{1}{N \bar{M}}\left[\sum_{i=1}^{n} m_{i} \bar{y}_{i}+\sum_{i=1}^{n} \frac{\bar{y}_{i}}{\bar{x}_{i}}\left(M_{i} \bar{X}_{i}-m_{i} \bar{x}_{i}\right)+\frac{\sum_{i=1}^{n} u_{i} \frac{\bar{y}_{i}}{\bar{x}_{i}} \bar{X}_{i}}{\sum_{i=1}^{n} u_{i} \bar{X}_{i}}\left(\bar{X}-\sum_{i=1}^{n} M_{i} \bar{X}_{i}\right)\right]=\frac{\sum_{i=1}^{n} u_{i} \frac{\bar{y}_{i}}{\bar{x}_{i}} \bar{X}_{i}}{\sum_{i=1}^{n} u_{i} \bar{X}_{i}} \bar{X} .
$$

It may be seen that $t_{p 5}$ is internally congruent estimator in the words of Sampford (1978), as the form of $t_{p 5}$ is equivalent to $T$. Incidentally $t_{p 5}$ happens to be Murthy's (1967) chain ratio estimator.

To the first order of approximation

$$
\begin{aligned}
\operatorname{MSE}\left(t_{p 5}\right)= & \left(\frac{1}{n}-\frac{1}{N}\right)\left(S_{b y}^{2}+R^{2} S_{b x}^{2}-2 R S_{b y x}\right) \\
& +\frac{1}{n N} \sum_{i=1}^{N} u_{i}^{2}\left(\frac{1}{m_{i}}-\frac{1}{M_{i}}\right)\left(S_{i y}^{2}+R_{i}^{2} S_{i x}^{2}-2 R_{i} S_{i y x}\right)
\end{aligned}
$$

\section{Comparison of predictive estimators}

When comparing the predictive estimators among themselves it is worthwhile to compare them with an estimator without auxiliary information, classical two stage ratio estimator and Smith's (1969) two stage ratio estimator. Thus an unbiased estimator in two stage sampling without using auxiliary information is given by

$$
\bar{y}=\frac{1}{n} \sum_{i=1}^{n} u_{i} \bar{y}_{i}
$$

with

$$
V(\bar{y})=\left(\frac{1}{n}-\frac{1}{N}\right) S_{b y}^{2}+\frac{1}{n N} \sum_{i=1}^{N} u_{i}^{2}\left(\frac{1}{m_{i}}-\frac{1}{M_{i}}\right) S_{i y}^{2} .
$$

Further the classical ratio estimator in two stage sampling using auxiliary information provided by $x$ is given by

$$
\bar{y}_{R}=\frac{\sum_{i=1}^{n} u_{i} \bar{y}_{i}}{\sum_{i=1}^{n} u_{i} \bar{x}_{i}} \bar{X}
$$

To the first order of approximation,

$$
\begin{aligned}
\operatorname{MSE}\left(\bar{y}_{R}\right)= & \left(\frac{1}{n}-\frac{1}{N}\right)\left(S_{b y}^{2}+R^{2} S_{b x}^{2}-2 R S_{b y x}\right) \\
& +\frac{1}{n N} \sum_{i=1}^{N} u_{i}^{2}\left(\frac{1}{m_{i}}-\frac{1}{M_{i}}\right)\left(S_{i y}^{2}+R^{2} S_{i x}^{2}-2 R S_{i y x}\right)
\end{aligned}
$$


Smith's (1969) multistage ratio estimator is given by

$$
\bar{y}_{R}^{\prime}=\frac{\sum_{i=1}^{n} u_{i} \bar{y}_{i}}{\sum_{i=1}^{n} u_{i} \bar{X}_{i}} \bar{X} .
$$

To the first order of approximation,

$$
\operatorname{MSE}\left(\bar{y}_{R}^{\prime}\right)=\left(\frac{1}{n}-\frac{1}{N}\right)\left(S_{b y}^{2}+R^{2} S_{b x}^{2}-2 R S_{b y x}\right)+\frac{1}{n N} \sum_{i=1}^{N} u_{i}^{2}\left(\frac{1}{m_{i}}-\frac{1}{M_{i}}\right) S_{i y}^{2}
$$

(i) The sufficient conditions when the predictive estimators $t_{p i}(i=1,2, \ldots, 5)$ would be more efficient than the unbiased estimator $\bar{y}$ are shown in Table 1.

Table 1. Comparison of predictive estimators with $\bar{y}$

\begin{tabular}{|c|l|}
\hline Predictive Estimators & Sufficient Conditions \\
\hline$t_{p 1}$ & (i) $\rho_{b y x}>\frac{1}{2} \frac{C_{b x}}{C_{b y}}$ \\
& (ii) $\beta_{i y x}>\frac{1}{2}\left[R-f\left(R-R_{i}\right)\right]$, for all $i \in U$ \\
\hline$t_{p 2}$ & (i) $\rho_{b y x}>\frac{1}{2} \frac{C_{b x}}{C_{b y}}$ \\
\hline$t_{p 3}$ & (ii) $\beta_{i y x}>\frac{1}{2}\left[R-f\left(R-f_{i} R_{i}\right)\right]$, for all $i \in U$ \\
\hline$t_{p 4}$ & (i) $\rho_{b y x}>\frac{1}{2} \frac{C_{b x}}{C_{b y}}$ \\
\hline & (ii) $\beta_{i y x}>\frac{1}{2} f R_{i}$, for all $i \in U$ \\
\hline$t_{p 5}$ & (i) $\rho_{b y x}>\frac{1}{2} \frac{C_{b x}}{C_{b y}}$ \\
& (ii) $\beta_{i y x}>\frac{1}{2} f f_{i} R_{i}$, for all $i \in U$ \\
\hline & (i) $\rho_{b y x}>\frac{1}{2} \frac{C_{b x}}{C_{b y}}$ \\
& (ii) $\beta_{i y x}>\frac{1}{2} R_{i}$, for all $i \in U$ \\
\hline
\end{tabular}

(ii) The sufficient conditions when the predictive estimators $t_{p i}(i=1,2, \ldots, 5)$ would be more efficient than $\bar{y}_{R}$ are shown in Table 2 . 
Table 2. Comparison of efficiencies predictive estimators $t_{p i}(i=1,2, \ldots, 5)$ with $\bar{y}_{R}$

\begin{tabular}{|c|l|}
\hline Predictive Estimators & Sufficient Conditions \\
\hline$t_{p 1}$ & $\begin{array}{l}\beta_{i y x}<\frac{1}{2}\left[2 R-f\left(R-R_{i}\right)\right] ; R>R_{i}, \text { for all } i \in U \text { or } \\
\beta_{i y x}>\frac{1}{2}\left[2 R-f\left(R-R_{i}\right)\right] ; R<R_{i}, \text { for all } i \in U\end{array}$ \\
\hline$t_{p 2}$ & $\begin{array}{l}\beta_{i y x}<\frac{1}{2}\left[2 R-f\left(R-f_{i} R_{i}\right)\right] ; R>f_{i} R_{i}, \text { for all } i \in U \text { or } \\
\beta_{i y x}>\frac{1}{2}\left[2 R-f\left(R-f_{i} R_{i}\right)\right] ; R<f_{i} R_{i}, \text { for all } i \in U\end{array}$ \\
\hline$t_{p 3}$ & $\begin{array}{l}\beta_{i y x}<\frac{1}{2}\left(R+f R_{i}\right) ; \text { if } R>f R_{i}, \text { for all } i \in U \text { or } \\
\beta_{i y x}>\frac{1}{2}\left(R+f R_{i}\right) ; \text { if } R<f R_{i}, \text { for all } i \in U\end{array}$ \\
\hline$t_{p 4}$ & $\begin{array}{l}\beta_{i y x}<\frac{1}{2}\left(R+f f_{i} R_{i}\right) ; R>f f_{i} R_{i}, \text { for all } i \in U \text { or } \\
\beta_{i y x}>\frac{1}{2}\left(R+f f_{i} R_{i}\right) ; R<f f_{i} R_{i}, \text { for all } i \in U\end{array}$ \\
\hline$t_{p 5}$ & $\begin{array}{l}\text { If } \beta_{i y x}<\frac{1}{2} R_{i}, R>R_{i}, \text { for all } i \in U \text { or } \\
\beta_{i y x}>\frac{1}{2} R_{i}, R_{i}>R, \text { for all } i \in U\end{array}$ \\
\hline
\end{tabular}

(iii) The sufficient conditions when the predictive estimators $t_{p i}(i=1,2, \ldots, 5)$ would be more efficient than $\bar{y}_{R}^{\prime}$ are shown in Table 3 .

Table 3. Comparison of predictive ratio type estimators with $\bar{y}_{R}^{\prime}$

\begin{tabular}{|c|l|}
\hline Predictive Estimators & Sufficient Conditions \\
\hline$t_{p 1}$ & $\beta_{i y x}>\frac{1}{2}\left[R-f\left(R-R_{i}\right)\right]$, for all $i \in U$ \\
\hline$t_{p 2}$ & $\beta_{i y x}>\frac{1}{2}\left[R-f\left(R-f_{i} R_{i}\right)\right]$, for all $i \in U$ \\
\hline$t_{p 3}$ & $\beta_{i y x}>\frac{1}{2} f R_{i}$, for all $i \in U$ \\
\hline$t_{p 4}$ & $\beta_{i y x}>\frac{1}{2} f f_{i} R_{i}$, for all $i \in U$ \\
\hline$t_{p 5}$ & $\beta_{i y x}>\frac{1}{2} R_{i}$, for all $i \in U$ \\
\hline
\end{tabular}

\section{Note}

(i) If $\beta_{i y x}>\frac{1}{2}\left[R-f\left(R-R_{i}\right)\right]$ for all $i \in U$, both $t_{p 1}$ and $t_{p 2}$ are more efficient than $\bar{y}_{R}^{\prime}$.

(ii) If $\beta_{i y x}>\frac{1}{2} R_{i}$, for all $i \in U, t_{p 3}, t_{p 4}$ and $t_{p 5}$ are more efficient than $\bar{y}_{R}^{\prime}$.

Now, it would be worthwhile to compare the internally congruent predictive estimator $t_{p 5}$ with non-congruent predictive estimators $-t_{p 1}, t_{p 2}, t_{p 3}$ and $t_{p 4}$. Thus, $t_{p 5}$ would be more efficient than 
(i) $t_{p 1}$, if for all $i \in U$,

$$
\begin{aligned}
& \beta_{i y x}<\frac{1}{2}\left[(1-f) R+(1+f) R_{i}\right], \quad R>R_{i}, \text { or } \\
& \beta_{i y x}>\frac{1}{2}\left[(1-f) R+(1+f) R_{i}\right], \quad R<R_{i} .
\end{aligned}
$$

(ii) $t_{p 2}$, if for all $i \in U$,

$$
\begin{aligned}
& \beta_{i y x}<\frac{1}{2}\left[(1-f) R+\left(1+f f_{i}\right) R_{i}\right], \quad R>\frac{1-f f_{i}}{1-f} R_{i}, \text { or } \\
& \beta_{i y x}>\frac{1}{2}\left[(1-f) R+\left(1+f f_{i}\right) R_{i}\right], \quad R<\frac{1-f f_{i}}{1-f} R_{i} .
\end{aligned}
$$

(iii) $t_{p 3}$, if for all $i \in U$,

$$
\beta_{i y x}<\frac{1}{2}(1+f) R_{i} .
$$

(iv) $t_{p 4}$, if for all $i \in U$,

$$
\beta_{i y x}<\frac{1}{2}\left(1+f f_{i}\right) R_{i} .
$$

\section{Numerical Illustration}

To illustrate efficiencies of different predictive and non-predictive estimators we consider 1971 population census data of Odisha (India).

The population consists of 104 blocks (ssu) divided into $N=15$ wards (fsu) of Berhampur City of Odisha (India). The number of blocks $\left(M_{i}\right)$ in 15 wards are 6, 6, 12, 5, 6, 6,10, 5, 6, 6, 6, 6, 6, 12 and 6. The two variables i.e., number of educated females and female population are denoted by $y$ and $x$ respectively. For comparison of asymptotic mean square errors (MSE) of $\bar{y}_{R}, \bar{y}_{R}^{\prime}, t_{p 1}, t_{p 2}, t_{p 3}$, $t_{p 4}$ and $t_{p 5}$ we consider first stage sample of size $n=5$. The sizes of the second stage samples $m_{i}$ $(i=1,2, \ldots, 15)$ are fixed at $2,2,4,2,2,2,3,2,3,3,2,2,2,4$, and 3 respectively.

The efficiencies of different estimators under comparison are compared in Table 4.

Table 4. Comparison of Efficiencies

\begin{tabular}{|c|c|c|}
\hline Estimator & MSE & Efficiency (\%) \\
\hline $\bar{y}$ & 302.9529 & 100 \\
\hline $\bar{y}_{R}$ & 222.0110 & 136.4585 \\
\hline $\bar{y}_{R}^{\prime}$ & 228.4768 & 132.5968 \\
\hline$t_{p 1}$ & 223.1162 & 135.7826 \\
\hline$t_{p 2}$ & 222.2914 & 136.2864 \\
\hline$t_{p 3}$ & 224.6903 & 134.8313 \\
\hline$t_{p 4}$ & 226.9665 & 133.4791 \\
\hline$t_{p 5}$ & 221.9455 & 136.4988 \\
\hline
\end{tabular}




\section{Conclusion}

The predictive ratio estimators including Murthy's chain ratio estimator (internally congruent predictive estimator) though logical in nature, are not uniformly more efficient than both the classical two stage ratio estimator $\bar{y}_{R}$ and Smith's two stage ratio estimator $\bar{y}_{R}^{\prime}$ and their superiority depends on certain sufficient conditions.

It is observed from the given illustration that $t_{p 5}$ (internally congruent predictive estimator) is the most efficient among the estimators under comparison, although the increase in efficiency is only marginal.

\section{References}

[1] Basu, D. (1971). An essay on the logical Foundations of Survey Sampling, Foundations of Statistical Inference, Part-I, Edited by V.P. Godambe and D.A. Sprott, Toronto, Holt Rinehart and Winston, 203242.

[2] Murthy, M.N. (1967). Sampling: Theory and Methods, Statistical Publishing Society, Calcutta, India.

[3] Panda, P. (1998). Some strategies in two stage sampling using auxiliary information, unpublished Ph.D. dissertation, Utkal University, India.

[4] Sampford, M.R. (1978). Predictive estimation and internal congruency, Contributions to survey sampling and Applied Statistics, Academic Press, New York, 29-39.

[5] Smith, T.M.F. (1969). A note on ratio estimate in multistage sampling, J.R.S.S., A, 132, 426-430.

[6] Sukhatme, P.V. and Sukhatme, B.V. and Asok, C. (1984). Sampling theory of surveys with applications, Ind. Soc. Ag. Stat., New Delhi. 